\title{
Legionnaires' disease in Europe: all quiet on the eastern front?
}

\author{
Julien Beauté, Emmanuel Robesyn and Birgitta de Jong, on behalf of the \\ European Legionnaires' Disease Surveillance Network
}

Affiliations: European Centre for Disease Prevention and Control (ECDC), Stockholm, Sweden.

Correspondence: J. Beauté, European Centre for Disease Prevention and Control (ECDC), Tomtebodavägen 11 , Stockholm 171 83, Sweden. E-mail: Julien.beautedecdc.europa.eu

0 @ERSpublications

ECDC draws attention to the under ascertainment of Legionnaires' disease in Eastern Europe http://ow.ly/p74HT

Almost 40 years ago, a large outbreak of pneumonia caused by a previously unidentified agent occurred at a hotel in Philadelphia (PA, USA), affecting 182 persons and causing the death of 29 [1]. All cases had attended a convention of the American Legion, a US wartime veteran service organisation. Both the causative agent of the outbreak and the disease were named after this event: Legionella spp. and Legionnaires' disease, respectively. Legionella spp. are Gram-negative bacteria found in freshwater environments worldwide and tend to contaminate and thrive in man-made water systems [2]. Legionnaires' disease is not transmitted from person to person. Infection occurs following inhalation of aerosols containing Legionella or aspiration of Legionella contaminated water, most commonly Legionella pneumophila serogroup 1 [2, 3], but other Legionella species have been documented (table 1) [4]. Legionnaires' disease usually presents as a severe pneumonia that may be accompanied by systemic symptoms. Known risk factors for Legionnaires' disease include increasing age, male sex, smoking, chronic lung disease, diabetes and various conditions associated with immunodeficiency $[5,6]$. Pontiac fever is a milder form of legionellosis, classically described as an influenza-like illness. Antibiotics with good intracellular action are most effective against any form of legionellosis. Azithromycin and levofloxacin are usually considered as the best first-line option [7, 8]. Mortality from Legionnaires' disease remains substantial $(\approx 10 \%)$, even in patients who receive appropriate treatment [9]. A summary of the characteristics of Legionnaires' disease and Pontiac fever are presented in table 2.

Since the first description of Legionnaires' disease, much of the attention has focused on large outbreaks because of their dramatic public health impact. About 10 years ago, the largest outbreak ever reported affected 449 cases in Murcia, Spain [10]. A more recent outbreak in 2012 comprised 50 confirmed cases in Edinburgh, UK [11]. In these two outbreaks and many others, a cooling tower was incriminated as the probable source. High-risk sites (cooling towers, spas, cruising ships, etc.) should be regularly checked for Legionella. Appropriate control measures may prevent a significant number of cases [12, 13]. Although of major public health relevance, these large outbreaks tend to eclipse the sporadic cases which form the majority of cases of Legionnaires' disease [14].

Infectious disease surveillance is an import tool in detecting unusual events, assessing trends and monitoring the impact of programmes [15-23]. Legionnaires' disease is notifiable in all 27 European Union countries, plus Iceland and Norway. In 1996, Legionnaires' disease surveillance at the European level was initiated by a network of motivated epidemiologists and microbiologists. Since 2010, this network has operated under the name of European Legionnaires' Disease Surveillance Network (ELDSNet), coordinated by the European Centre for Disease Prevention and Control (ECDC) in Stockholm, Sweden. Two distinct

Received: May 242013 | Accepted after revision: June 282013

Conflict of interest: None declared.

Copyright @ERS 2013 
TABLE 1 Summary of agent, mechanism of transmission and risk factors

All cases

Mostly Legionella pneumophila sg1

Cooling towers

Hot and cold water systems

Recreational pools and spas

Mechanism of transmission

Risk factors

(environmental)

Risk factors (hosts)

Aspiration possible

Warm season Poor water temperature control
Setting-related specificities

Legionella longbeachae in potting soils (community acquired)

Potting and compost (community acquired)

Respiratory therapy equipment (healthcare associated)

Aspiration or direct instillation into the lung during respiratory tract manipulations (healthcare associated) Wound infection (healthcare-associated)

Poorly designed and poorly maintained water systems

$$
\begin{gathered}
\text { Increasing age } \\
\text { Male } \\
\text { Smoking }
\end{gathered}
$$

Chronic lung disease

Diabetes

Conditions associated with immunodeficiency
Accommodation with seasonal/irregular water use (travel associated)

Recent surgery (healthcare associated) Intubation (healthcare associated) Aspiration (healthcare associated)

Data from [12].

Legionnaires' disease surveillance systems are currently in place. One is based on annual passive reporting of all Legionnaires' disease cases notified nationally, the other on the daily reporting of cases of travelassociated Legionnaires' disease (TALD). The latter scheme aims at identifying and containing clusters linked to stays in the same accommodation, which otherwise may not be detected at a national level. The success of the European TALD surveillance, as well as in triggering preventive measures in tourist accommodation, has now been well established, and each year nearly half of the clusters detected would have probably remained unnoticed without the network because the cases associated were reported by different countries $[24,25]$.

The surveillance of all Legionnaires' disease cases in Europe has provided interesting insights. First, after a period of steady increase at the European level, the number and rate of annually reported cases has

\section{TABLE 2 Main characteristics and treatment of Legionnaires' disease and Pontiac fever}

\section{Legionnaires' disease}

\section{Attack rate \\ Incubation \\ Symptoms}

Low $(<1 \%)$

Usually 2-10 days

Pneumonia

\section{Chest radiograph \\ Laboratory findings \\ Duration \\ Diagnosis}

\section{Treatment} Case-fatality rate

Notification at European level
Non-productive cough

Fever $>39^{\circ} \mathrm{C}$

Extrapulmonary symptoms

Gastrointestinal symptoms (diarrhoea)

Neurological disorders (confusion and delirium)

Abnormal, but nonspecific

Renal and hepatic dysfunction Weeks

Urinary antigen test (most commonly used) Culture (gold standard)

Other blood tests (less frequently used)

Azithromycin or levofloxacin

$$
\approx 10 \%
$$

Mandatory
Pontiac fever

$$
\text { High }
$$

Influenza-like illness

Normal

Normal

2-5 days

Urinary antigen test

Symptomatic

No deaths

None

Data from [12]. 
plateaued or followed a slow increasing trend from 2005 to 2010 [14]. This may suggest that national surveillance schemes have reached their maximum detection capacity under current diagnostic conditions, at least in the countries reporting most cases (six countries accounted for $\approx 85 \%$ of all cases in 2011 [24]). One survey aimed at estimating the annual number of tests performed in each European country which could help understand why the majority of reported cases are concentrated in a few countries. Secondly, there are large differences in Legionnaires' disease notification rates between European Union countries (fig. 1), which cannot be fully explained by differences in local risk and seem to point to important disparities in awareness among clinicians, diagnostic capability or capacity, and reporting across countries. This is especially striking in eastern and south-eastern European countries where the numbers of cases reported are far below the European Union average of approximately 10 per million population [24-26]. In 2011, the notification rate of community-acquired Legionnaires' disease was below one per million in Bulgaria, the Czech Republic, Greece, Romania and Slovakia (fig. 1). A recent study using tourism patterns as denominators also suggested that under-ascertainment was substantial in Greece [27]. Thirdly, the disease's seasonality, with most cases reported during the warm season, is highly suggestive of an influence of environmental conditions on Legionnaires' disease incidence. This was already suggested by previous studies [28-30], even though the link between weather patterns and Legionnaires' disease incidence remains to be fully elicited. Finally, the urinary antigen test has now become by far the most used diagnostic test for Legionnaires' disease. Since this test only detects L. pneumophila serogroup 1, some cases due to other species may be missed. The reference method (culture) was used in only $12 \%$ of all cases reported in 2011 in Europe with substantial differences across countries [24].

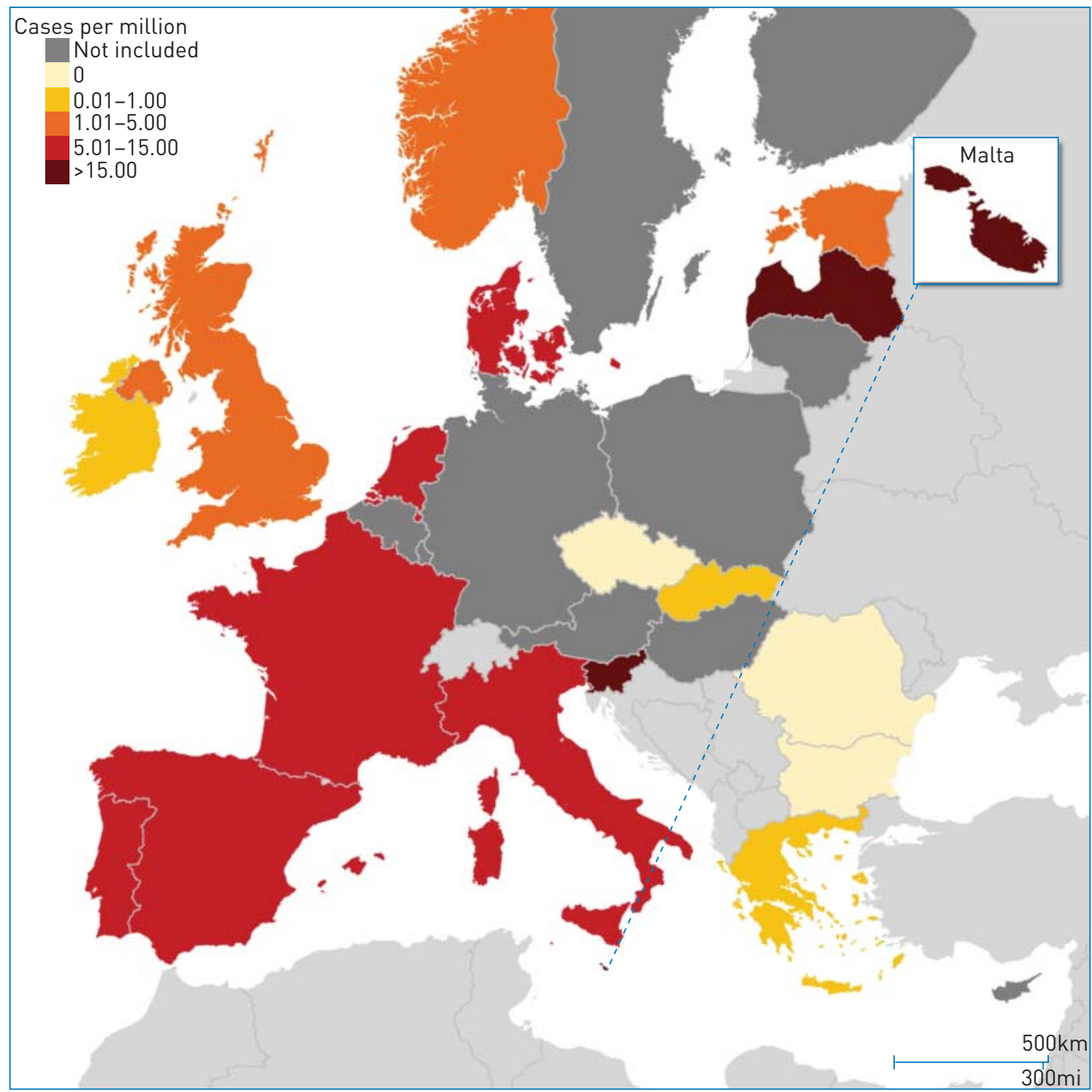

FIGURE 12011 notification rates of community-acquired Legionnaires' disease, according to the European Union/ European Economic Area $(n=2642)$. Countries with $>25 \%$ of infection settings reported as unknown were not included. 
These findings have important implications for both clinicians and public health professionals. First, clinicians should not consider Legionnaires' disease as an unusual, exotic disease. Prospective studies have suggested that $L$. pneumophila was the causative pathogen in $2 \%$ of outpatients, $5 \%$ of hospitalised patients and $8 \%$ of intensive care unit patients with community-acquired pneumonia (CAP) [31]. Secondly, Legionnaires' disease is not merely a disease affecting southern countries. High notification rates of community-acquired Legionnaires' disease have been observed in northern European countries, such as Denmark or Latvia (11 and 18 cases/million inhabitants in 2011, respectively [24]). Finally, if routine testing for Legionnaires' disease has not been recommended for all outpatients with CAP for reasons of costeffectiveness [32], testing should be considered in any patient with unexplained pneumonia and risk factors for Legionnaires' disease and should probably be expanded during the warm season. Apart from a quicker and more effective antimicrobial choice, this will allow for a better detection and control of possible sources of new infections.

A better understanding of the risk for Legionnaires' disease would allow us to better target preventive measures and improve clinician awareness. Countries with unrealistically low notification rates may need support in identifying and addressing local causes of under ascertainment, but also in improving prevention and control of the disease. Due to this, the ECDC organises annual Legionnaires' disease training courses for public health experts in the field of epidemiology, microbiology and environmental health. However, clinical societies, such as the European Respiratory Society, are much better placed to increase awareness among clinicians, be it through education and training, or through promotion of local studies (e.g. crosssectional studies at major university hospitals in countries with very low notification rates). A close collaboration between public health, clinicians and microbiologists is of utmost importance for further improving the surveillance and consequently the prevention of Legionnaires' disease and its high morbidity and mortality.

\section{References}

1 Fraser DW, Tsai TR, Orenstein W, et al. Legionnaires' disease: description of an epidemic of pneumonia. $N$ Engl J Med 1977; 297: 1189-1197.

2 Fields BS, Benson RF, Besser RE. Legionella and Legionnaires' disease: 25 years of investigation. Clin Microbiol Rev 2002; 15: 506-526.

Carratalà J, Garcia-Vidal C. An update on Legionella. Curr Opin Infect Dis 2010; 23: 152-157.

De Jong B, Zucs P. Legionella, springtime and potting soils. Euro Surveill 2010; 15: 19497.

Marston BJ, Lipman HB, Breiman RF. Surveillance for Legionnaires' disease. Risk factors for morbidity and mortality. Arch Intern Med 1994; 154: 2417-2422.

6 Ginevra C, Duclos A, Vanhems P, et al. Host-related risk factors and clinical features of community-acquired Legionnaires disease due to the Paris and Lorraine endemic strains, 1998-2007, France. Clin Infect Dis 2009; 49: 184-191.

7 Woodhead M, Blasi F, Ewig S, et al. Guidelines for the management of adult lower respiratory tract infections - full version. Clin Microbiol Infect. 2011; 17: Suppl. 6, E1-E59.

8 Pedro-Botet ML, Yu VL. Treatment strategies for Legionella infection. Expert Opin Pharmacother 2009; 10: 1109-1121.

9 Chidiac C, Che D, Pires-Cronenberger S, et al. Factors associated with hospital mortality in community-acquired legionellosis in France. Eur Respir J 2012; 39: 963-970.

10 García-Fulgueiras A, Navarro C, Fenoll D, et al. Legionnaires' disease outbreak in Murcia, Spain. Emerging Infect Dis 2003; 9: 915-921.

11 McCormick D, Thorn S, Milne D, et al. Public health response to an outbreak of Legionnaires' disease in Edinburgh, United Kingdom, June 2012. Euro Surveill 2012; 17: 20216.

12 Chartier Y, Lee JV, Pond K, et al., eds. Legionella and the Prevention of Legionellosis. Geneva, World Health Organization, 2007.

13 Azara A, Piana A, Sotgiu G, et al. Prevalence study of Legionella spp. contamination in ferries and cruise ships. BMC Public Health 2006; 6: 100.

14 Beauté J, Zucs P, de Jong B. Legionnaires' disease in Europe, 2009-2010. Euro Surveill 2013; 18: 20417.

15 Zignol M, Sismanidis C, Falzon D, et al. Multidrug-resistant tuberculosis in children: evidence from global surveillance. Eur Respir J 2013; 42: 701-707.

16 Van Hest R, Kodmon C, Verver S, et al. Tuberculosis treatment outcome monitoring in European Union countries: systematic review. Eur Respir J 2012; 41: 635-643.

17 Van der Werf MJ, Blasi F, Giesecke J, et al. Lessons learnt in Europe on tuberculosis surveillance, outbreaks and BCG vaccination in 2011. Eur Respir J 2013; 41: 767-771.

18 Pontali E, Pasticci MB, Matteelli A, et al. Tuberculosis and HIV co-infection: do we have a surveillance system in Europe? Eur Respir J 2011; 38: 1258-1260.

19 Pimpin L, Drumright LN, Kruijshaar ME, et al. Tuberculosis and HIV co-infection in European Union and European Economic Area countries. Eur Respir J 2011; 38: 1382-1392.

20 Migliori GB, Zellweger JP, Abubakar I, et al. European Union standards for tuberculosis Care. Eur Respir J 2012; 39: 807-819.

21 Migliori GB, Sotgiu G, D'Ambrosio L, et al. TB and MDR/XDR-TB in European Union and European Economic Area countries: managed or mismanaged? Eur Respir J 2012; 39: 619-625.

22 Kruijshaar ME, Pimpin L, Abubakar I, et al. The burden of TB-HIV in the EU: how much do we know? A survey of surveillance practices and results. Eur Respir J 2011; 38: 1374-1381. 
23 Jenkins HE, Plesca V, Ciobanu A, et al. Assessing spatial heterogeneity of MDR-TB in a high burden country. Eur Respir J 2012 [in press DOI: 10.1183/09031936.00111812].

24 European Centre for Disease Prevention and Control. Legionnaires' disease surveillance in Europe, 2011. Stockholm, ECDC, 2013.

25 European Centre for Disease Prevention and Control. Legionnaires' disease surveillance in Europe, 2010. Stockholm, ECDC, 2012.

26 European Centre for Disease Prevention and Control. Legionnaires' disease surveillance in Europe, 2009. Stockholm, ECDC, 2011.

27 Beauté J, Zucs P, de Jong B. Risk for travel-associated Legionnaires’ Disease, Europe, 2009. Emerg Infect Dis 2012; 18: $1811-1816$.

28 Ricketts KD, Charlett A, Gelb D, et al. Weather patterns and Legionnaires' disease: a meteorological study. Epidemiol Infect 2009; 137: 1003-1012.

29 Hicks LA, Rose CE Jr, Fields BS, et al. Increased rainfall is associated with increased risk for legionellosis. Epidemiol Infect 2007; 135: 811-817.

30 Fisman DN, Lim S, Wellenius GA, et al. It's not the heat, it's the humidity: wet weather increases legionellosis risk in the greater Philadelphia metropolitan area. J Infect Dis 2005; 192: 2066-2073.

31 Woodhead M. Community-acquired pneumonia in Europe: causative pathogens and resistance patterns. Eur Respir J 2002; 20: Suppl. 36, 20s-27s.

32 File TM. Community-acquired pneumonia. Lancet 2003; 362: 1991-2001. 